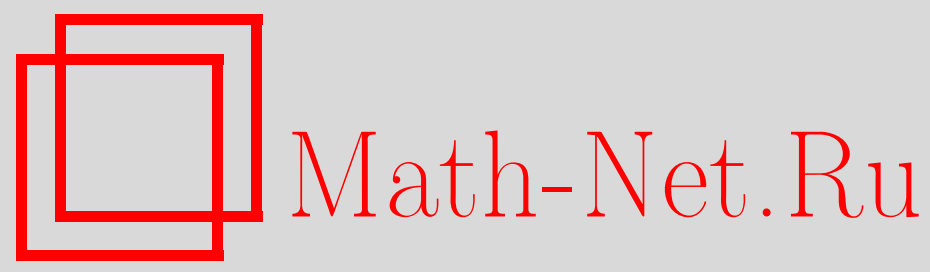

Т. Г. Хессами Пилеруд, Оценки снизу линейных форм от значений некоторых гипергеометрических функций, $M a$ тем. заметки, 2000, том 67, выпуск 3, 441-451

DOI: https://doi.org/10.4213/mzm856

Использование Общероссийского математического портала Math-Net.Ru подразумевает, что вы прочитали и согласны с пользовательским соглашением http://www.mathnet.ru/rus/agreement

Параметры загрузки:

IP : 52.90 .164 .192

26 апреля 2023 г., $13: 27: 28$

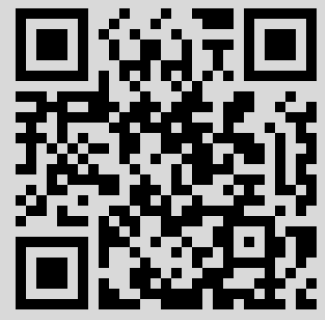




\section{ОЦЕНКИ СНИЗУ ЛИНЕЙНЫХ ФОРМ ОТ ЗНАЧЕНИЙ НЕКОТОРЫХ ГИПЕРГЕОМЕТРИЧЕСКИХ ФУНКЦИЙ}

\section{Т. Г. Хессами Пилеруд}

В работе с помощью аппроксимаций Паде первого рода получена оценка снизу для модуля линейной формы с целыми коэффициентами от значений некоторых гипергеометрических функций в рациональной точке. Эта оценка учитьвает рост всех коэффициентов линейной формы.

Библиография: 7 названий.

В данной работе изучаются арифметические свойства значений некоторых гипергеометрических функций в рациональной точке. С помощью приближений Паде первого рода для таких функций получены оценки снизу линейных форм от значений гипергеометрических функций, зависящие от всех коэффициентов формы.

Для $j=1, \ldots, m$ положим

$$
f_{j}(z)=1+\sum_{\nu=1}^{\infty} \frac{\left(\alpha_{1}\right)_{\nu}}{\left(\beta_{1}\right)_{\nu}} \cdot \frac{z^{\nu}}{\left(\beta_{j}+\nu\right)}, \quad|z|<1
$$

где $(\alpha)_{0}=1$ и $(\alpha)_{\nu}=\alpha(\alpha+1) \cdots\left(\alpha_{1}+\nu-1\right)$ при $\nu \geqslant 1 ; \alpha_{1}, \beta_{1}, \ldots, \beta_{m}$ - некоторый набор рациональных чисел. В дальнейшем через $\operatorname{den} \xi \in \mathbb{N}$ обозначается знаменатель несократимой дроби $\xi$. Для $k \in \mathbb{N}$ определим

$$
h(k)=\frac{k}{\varphi(k)} \sum_{\substack{r=1 \\(r, k)=1}}^{k} \frac{1}{r}
$$

где $\varphi$ - функция Эйлера.

Теорема 1. Пусть числа $\alpha_{1}, \beta_{1}, \ldots, \beta_{m} \in \mathbb{Q} u \beta_{i}-\beta_{j}, 1+\beta_{i}-\alpha_{1}(i, j=1, \ldots, m$, $i \neq j$ ) отличны от нуля и отричательных иелых,

$$
\begin{gathered}
a_{1}=\operatorname{den} \alpha_{1}, \quad b_{j}=\operatorname{den} \beta_{j}, \\
b_{i, j}=\operatorname{den}\left(\beta_{i}-\beta_{j}\right), \quad a_{1, j}=\operatorname{den}\left(\alpha_{1}-\beta_{j}\right), \quad i, j=1, \ldots, m, \quad i \neq j, \\
G=\sum_{k=1}^{m}\left(h\left(a_{1, k}\right)+h\left(b_{k}\right)+\sum_{\substack{l=1 \\
l \neq k}}^{m} h\left(b_{l, k}\right)\right), \\
F=2\left(\ln a_{1}+\sum_{\substack{p \mid a_{1} \\
p \text { простое }}} \frac{\ln p}{p-1}\right)+\sum_{j=1}^{m}\left(\ln b_{j}+\sum_{\substack{p \mid b_{j} \\
p \text { простое }}} \frac{\ln p}{p-1}\right)+G .
\end{gathered}
$$


Пусть, далее, $b \in \mathbb{Z}, a \in \mathbb{N}$ и выполнено неравенство

$$
|b|>a^{m+1} \cdot \exp \{(m-1)(m \ln 4 m+\ln 4)+m F\} .
$$

Тогда существует постоянная $c=c\left(\alpha_{1}, \beta_{1}, \ldots, \beta_{m}, a, b\right)>0$ такая, что для любых челых чисел $x_{0}, x_{1}, \ldots, x_{m}$, в совокупности отличных от нуля, имеет место неравенство

$$
\left|x_{0}+\sum_{i=1}^{m} x_{i} f_{i}\left(\frac{a}{b}\right)\right|>c\left(\bar{x}_{1} \cdots \bar{x}_{m}\right)^{-1} \cdot \bar{x}_{1}^{-\delta},
$$

əде $\bar{x}_{i} \geqslant \max \left\{1,\left|x_{i}\right|\right\}(i=1, \ldots, m), \bar{x}_{1}=\max \left\{\bar{x}_{1}, \ldots, \bar{x}_{m}\right\}$,

$$
\delta=\frac{m(m+1) \ln a+m(m+1) F+m^{3} \ln 4 m+m^{2} \ln 4}{\ln |b|-(m+1) \ln a-m F-(m-1)(m \ln 4 m+\ln 4)} .
$$

СлЕДСТВИЕ 1. Пусть $b \in \mathbb{Z}, a \in \mathbb{N}, \varepsilon>0 u F-$ то жее, ито в теореме 1 ,

$$
|b|^{\varepsilon /(m+\varepsilon)}>a^{m+1} \cdot \exp \left\{m^{2} \ln 4 m+(m+1)(F+\ln 4)\right\} .
$$

Тогда для любого нетривиального набора челых чисел $x_{0}, x_{1}, \ldots, x_{m}$ и чисел $\bar{x}_{i} \geqslant$ $\max \left\{1,\left|x_{i}\right|\right\}(i=1, \ldots, m)$, удовлетворяющих условиям

$$
\bar{x}_{1}=\max \left\{\bar{x}_{1}, \ldots, \bar{x}_{m}\right\}, \quad \bar{x}_{1} \geqslant X_{0}=X_{0}\left(\alpha_{1}, \beta_{1}, \ldots, \beta_{m}, a, b\right)>0,
$$

справедливо неравенство

$$
\left|x_{0}+\sum_{i=1}^{m} x_{i} f_{i}\left(\frac{a}{b}\right)\right|>\left(\bar{x}_{1} \cdots \bar{x}_{m}\right)^{-1} \cdot \bar{x}_{1}^{-\varepsilon} .
$$

Принцип Дирихле показьвает, что уточнение последнего неравенства возможно лишь за счет величины $\varepsilon$.

Первая оценка линейной формы, зависящая от всех коэффициентов, для чисел $e^{\alpha_{i}}$, $\alpha_{i} \in \mathbb{Q}, i=1, \ldots, m$, была получена А. Бейкером в [1].

Для значений некоторых $G$-функций оценки такого типа с помощью явных функциональных конструкций приближений получены пока только в работах [2]-[4]. Теорема 1 обобщает результат работы [2].

Оценки линейных форм, зависящие от всех коэффициентов, от значений функций $f_{j}(z)$ можно получать и с помощью метода Зигеля-Шидловского [5]. Однако эффективное построение приближающих форм дает лучшие результаты.

Перейдем к доказательству теоремы.

1. Построение приближающих форм. Пусть $\alpha_{1}, \beta_{1}, \ldots, \beta_{m} \in \mathbb{Q} \backslash\{0,-1,-2, \ldots\}$ и

$$
\beta_{i}-\beta_{j} \neq 0,-1,-2, \ldots, \quad 1+\beta_{i}-\alpha_{1} \neq 0,-1,-2, \ldots, \quad i, j=1, \ldots, m, \quad i \neq j .
$$

Пусть далее $n_{1}, \ldots, n_{m}$ - натуральные числа,

$$
n_{1}=\max \left\{n_{1}, \ldots, n_{m}\right\}, \quad N+1=\sum_{i=1}^{m}\left(n_{i}+1\right), \quad \boldsymbol{n}=\left(n_{1}, \ldots, n_{m}\right) .
$$

Положим

$$
\begin{aligned}
& R_{\boldsymbol{n}}(\nu)=\frac{(\nu-1)(\nu-2) \cdots(\nu-N)}{\left(\beta_{1}+\nu\right)_{n_{1}+1} \cdots\left(\beta_{m}+\nu\right)_{n_{m}+1}} \\
& F_{\boldsymbol{n}}(z)=\sum_{\nu=1}^{\infty} \frac{\left(\alpha_{1}\right)_{\nu}}{\left(\beta_{1}\right)_{\nu}} R_{\boldsymbol{n}}(\nu) z^{-\nu}
\end{aligned}
$$


Лемма 1. Существуют рачиональные числа $\omega_{j, s}\left(j=1, \ldots, m ; s=0,1, \ldots, n_{j}\right)$, для которых тождественно по у выполнено равенство

$$
R_{\boldsymbol{n}}(\nu)=\sum_{j=1}^{m} \sum_{s=0}^{n_{j}} \omega_{j, s} \frac{\left(\alpha_{1}+\nu\right)_{s}}{\left(\beta_{1}+\nu\right)_{s}\left(\beta_{j}+\nu+s\right)} .
$$

ДокАЗАТЕЛЬСтво. Умножим обе части (5) на знаменатель $R_{\boldsymbol{n}}(\nu)$; тогда задача сведется к доказательству существования чисел $\omega_{j, s} \in \mathbb{Q}\left(j=1, \ldots, m ; s=0,1, \ldots, n_{j}\right)$ таких, что тождественно по $\nu$

$$
(\nu-1) \cdots(\nu-N)=\sum_{j=1}^{m} \sum_{s=0}^{n_{j}} \omega_{j, s}\left(\alpha_{1}+\nu\right)_{s} \cdot T_{j, s}(\nu),
$$

где $T_{j, s}(\nu)$ - многочлен степени $N-s$ равньй

$$
T_{j, s}(\nu)=\frac{\left(\beta_{1}+\nu\right)_{n_{1}+1} \cdots\left(\beta_{m}+\nu\right)_{n_{m}+1}}{\left(\beta_{1}+\nu\right)_{s}\left(\beta_{j}+\nu+s\right)} .
$$

Подставляя в (6) последовательно $\nu=1,2, \ldots, N$, получим систему $N$ линейных однородных уравнений относительно $N+1$ неизвестных $\omega_{j, s}$, которая имеет ненулевое решение с точностью до нормировочного множителя, которьй определим ниже. Тогда для выбранного ненулевого решения

$$
\sum_{j=1}^{m} \sum_{s=0}^{n_{j}} \omega_{j, s}\left(\alpha_{1}+\nu\right)_{s} \cdot T_{j, s}(\nu)=T(\nu),
$$

где $T(\nu)$ - некоторьй многочлен степени не вьше $N$.

Если $\operatorname{deg} T(\nu)<N$, то $T(\nu) \equiv 0$, и

$$
\sum_{j=1}^{m} \sum_{s=0}^{n_{j}} \omega_{j, s}\left(\alpha_{1}+\nu\right)_{s} \cdot T_{j, s}(\nu) \equiv 0 .
$$

Заметим, что при $1 \leqslant j \leqslant m, 0 \leqslant s \leqslant n_{j}$ в силу (7) и $(2) \beta_{j}+\nu+s$ делит $T_{j^{\prime}, s^{\prime}}(\nu)$ при всех допустимых значениях $\left(j^{\prime}, s^{\prime}\right) \neq(j, s)$ и не делит $\left(\alpha_{1}+\nu\right)_{s} T_{j, s}(\nu)$. Тогда из $(8)$ следует, что $\omega_{j, s}=0\left(1 \leqslant j \leqslant m, 0 \leqslant s \leqslant n_{j}\right)$, а это невозможно.

Таким образом, $\operatorname{deg} T(\nu)=N$ и при соответствуюшем выборе нормировочного множителя $T(\nu)=(\nu-1) \cdots(\nu-N)$. Лемма доказана.

Лемма 2. Справедливо равенство

$$
F_{\boldsymbol{n}}(z)=\sum_{j=1}^{m} P_{\boldsymbol{n}, j}(z) \cdot f_{j}\left(z^{-1}\right)-P_{\boldsymbol{n}, 0}(z),
$$

əде

$$
\begin{aligned}
& P_{\boldsymbol{n}, j}(z)=\sum_{s=0}^{n_{j}} \omega_{j, s} z^{s}, \quad 1 \leqslant j \leqslant m \\
& P_{\boldsymbol{n}, 0}(z)=\sum_{i=1}^{m} \sum_{s=0}^{n_{i}} \omega_{j, s} z^{s}\left(1+\sum_{\nu=1}^{s} \frac{\left(\alpha_{1}\right)_{\nu} z^{-\nu}}{\left(\beta_{1}\right)_{\nu}\left(\beta_{i}+\nu\right)}\right) .
\end{aligned}
$$


ДокаЗАТЕЛЬСТво. Из (4) и (5) имеем

$$
\begin{aligned}
F_{\boldsymbol{n}}(z) & =\sum_{j=1}^{m} \sum_{s=0}^{n_{j}} \omega_{j, s} z^{s} \cdot\left(\sum_{\nu=1}^{\infty} \frac{\left(\alpha_{1}\right)_{\nu+s} z^{-\nu-s}}{\left(\beta_{1}\right)_{\nu+s}\left(\beta_{j}+\nu+s\right)}\right) \\
& =\sum_{j=1}^{m} \sum_{s=0}^{n_{j}} \omega_{j, s} z^{s} \cdot\left(f_{j}\left(z^{-1}\right)-1-\sum_{k=1}^{s} \frac{\left(\alpha_{1}\right)_{k} z^{-k}}{\left(\beta_{1}\right)_{k}\left(\beta_{j}+k\right)}\right) \\
& =\sum_{j=1}^{m} P_{\boldsymbol{n}, j}(z) \cdot f_{j}\left(z^{-1}\right)-P_{\boldsymbol{n}, 0}(z),
\end{aligned}
$$

и лемма доказана.

Лемма 3. Существуют положительные числа $c_{1}, c_{2}$, зависящие только от $\alpha_{1}$, $\beta_{1}, \ldots, \beta_{m}$, такие, что при $|z| \geqslant 2$ и всех $\boldsymbol{n} \in \mathbb{N}^{m}$ с условием $\min \left\{n_{1}, \ldots, n_{m}\right\} \geqslant c_{1}$ справедливо неравенство

$$
\left|F_{n}(z)\right| \leqslant N^{c_{2}}|z|^{-N-1} .
$$

ДокАЗАТЕЛЬСТво. Из (3) и (4) получаем

$$
F_{n}(z)=\frac{\Gamma\left(\beta_{1}\right)}{\Gamma\left(\alpha_{1}\right)} \sum_{\nu=N+1}^{\infty} \frac{\Gamma\left(\alpha_{1}+\nu\right)}{\Gamma\left(\beta_{1}+\nu\right)} \frac{\Gamma(\nu)}{\Gamma(\nu-N)} \prod_{i=1}^{m} \frac{\Gamma\left(\beta_{i}+\nu\right)}{\Gamma\left(\beta_{i}+\nu+n_{i}+1\right)} z^{-\nu} .
$$

Всюду в дальнейшем $c_{3}, c_{4}, \ldots$ - некоторые положительные константы, зависяшие только от $\alpha_{1}, \beta_{1}, \ldots, \beta_{m}$.

По формуле Стирлинга имеем

$$
\begin{gathered}
\frac{\Gamma\left(\alpha_{1}+\nu\right)}{\Gamma\left(\beta_{1}+\nu\right)} \leqslant c_{3} \cdot \nu^{\alpha_{1}-\beta_{1}} \\
\frac{\Gamma(\nu)}{\Gamma(\nu-N)} \leqslant c_{4} \cdot \exp \left\{N \ln \nu-\left(\nu-N-\frac{1}{2}\right) \ln \left(1-\frac{N}{\nu}\right)-N\right\} .
\end{gathered}
$$

С учетом того, что

$$
\begin{gathered}
-\left(\nu-N-\frac{1}{2}\right) \ln \left(1-\frac{N}{\nu}\right)=\left(\nu-N-\frac{1}{2}\right) \sum_{r=1}^{\infty} \frac{N^{r}}{r \nu^{r}} \\
\leqslant \sum_{r=1}^{\infty} \frac{N^{r}}{r \nu^{r-1}}-\sum_{r=1}^{\infty} \frac{N^{r+1}}{r \nu^{r}}=N-\sum_{r=1}^{\infty} \frac{N^{r+1}}{r(r+1) \nu^{r}}<N,
\end{gathered}
$$

находим

$$
\frac{\Gamma(\nu)}{\Gamma(\nu-N)}<c_{4} \cdot \nu^{N}
$$

Далее

$\frac{\Gamma\left(\beta_{i}+\nu\right)}{\Gamma\left(\beta_{i}+\nu+n_{i}+1\right)} \leqslant c_{5} \exp \left\{\left(-1-n_{i}\right) \ln \nu+n_{i}+1-\left(\beta_{i}+\nu+n_{i}+1\right) \ln \left(1+\frac{\beta_{i}+n_{i}+1}{\nu}\right)\right\}$. 
Tak кaK

$$
\ln \left(1+\frac{\beta_{i}+n_{i}+1}{\nu}\right) \geqslant \frac{\beta_{i}+n_{i}+1}{\nu}-\frac{\left(\beta_{i}+n_{i}+1\right)^{2}}{2 \nu^{2}}
$$

при достаточно большом $\min \left\{n_{1}, \ldots, n_{m}\right\}$ выполнено

$$
\begin{aligned}
& -\left(\beta_{i}+\nu+n_{i}+1\right) \ln \left(1+\frac{\beta_{i}+n_{i}+1}{\nu}\right) \\
& \quad \leqslant-\beta_{i}-n_{i}-1+\frac{\left(\beta_{i}+n_{i}+1\right)^{2}}{2 \nu}\left(-1+\frac{\beta_{i}+n_{i}+1}{\nu}\right) \leqslant-\beta_{i}-n_{i}-1
\end{aligned}
$$

и

$$
\frac{\Gamma\left(\beta_{i}+\nu\right)}{\Gamma\left(\beta_{i}+\nu+n_{i}+1\right)}<c_{6} \cdot \nu^{-n_{i}-1}
$$

Окончательно из (9)-(12) при $|z| \geqslant 2$ получаем

$$
\left|F_{n}(z)\right| \leqslant c_{7} \sum_{\nu=N+1}^{\infty} \nu^{\alpha_{1}-\beta_{1}-1}|z|^{-\nu}<|z|^{-N-1} N^{c_{8}} \sum_{k=1}^{\infty} \frac{k^{\alpha_{1}-\beta_{1}}}{2^{k}}=N^{c_{9}}|z|^{-N-1}
$$

и лемма доказана.

Пусть $\Theta$ - замкнутьй, положительно-ориентированный, кусочно-гладкий контур, охватывающий точки $-\beta_{1}-s,-\beta_{j}-s-1, \ldots,-\beta_{j}-n_{j}(j=1, \ldots, m)$ и не содержащий внутри себя остальных точек вида $-\beta_{k}-l,-\alpha_{1}-r$, отличных от перечисленных. Для $1 \leqslant j \leqslant m, 0 \leqslant s \leqslant n_{j}$ пусть число $\rho_{j, s} \in(0,1 / 2]$ настолько мало, что внутри окружности $\left|\beta_{j}+\zeta+s\right|=\rho_{j, s}$ нет других точек вида $-\beta_{k}-l,-\alpha_{1}-r$, отличных от $-\beta_{j}-s$.

Тогда из (5) легко получаем следующие интегральные представления для чисел $\omega_{j, s}$ :

$$
\begin{aligned}
\omega_{j, s}= & \frac{1}{2 \pi i} \oint_{\left|\beta_{j}+\zeta+s\right|=\rho_{j, s}} R_{n}(\zeta) \frac{\left(\beta_{1}+\zeta\right)_{s}}{\left(\alpha_{1}+\zeta\right)_{s}} d \zeta, \quad 2 \leqslant j \leqslant m, \quad 0 \leqslant s \leqslant n_{j}, \\
\omega_{1, s}= & \begin{array}{ll}
\frac{1}{2 \pi i} \oint_{\left|\beta_{1}+\zeta+s\right|=\rho_{1, s}} R_{n}(\zeta) \frac{\left(\beta_{1}+\zeta\right)_{s}}{\left(\alpha_{1}+\zeta\right)_{s}} d \zeta, & \text { если } \alpha_{1}=\beta_{1}, \\
\frac{\alpha_{1}-\beta_{1}}{2 \pi i} \oint_{\Theta} R_{n}(\zeta) \frac{\left(\beta_{1}+\zeta\right)_{s}}{\left(\alpha_{1}+\zeta\right)_{s+1}} d \zeta, & \text { если } \alpha_{1} \neq \beta_{1},
\end{array}
\end{aligned}
$$

(в формулах (13), (14) число $i$ является мнимой единицей).

ЛЕмма 4. При $|z|>1$ и всех $\boldsymbol{n} \in \mathbb{N}^{m}$ таких, что $n_{1}>1$ справедливы неравенства

$$
\left|P_{n, j}(z)\right| \leqslant n_{1}^{c_{10}} e^{(m \ln 4 m+\ln 4) n_{1}}|z|^{n_{j}}
$$

$n p u 1 \leqslant j \leqslant m, 0 \leqslant s \leqslant n_{j}$. 
ДокАЗАТЕЛЬСТво. Оценим функцию

$$
R_{\boldsymbol{n}}(\zeta) \frac{\left(\beta_{1}+\zeta\right)_{s}}{\left(\alpha_{1}+\zeta\right)_{s}}
$$

на окружности $\left|\beta_{j}+\zeta+s\right|=\rho_{j, s}$ для всех допустимых значений $j$ и $s$ в (13) и для случая

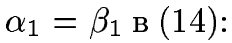

$$
\left|R_{\boldsymbol{n}}(\zeta) \frac{\left(\beta_{1}+\zeta\right)_{s}}{\left(\alpha_{1}+\zeta\right)_{s}}\right| \leqslant \frac{(|\zeta|+1)(|\zeta|+2) \cdots(|\zeta|+N)}{\left|\left(\beta_{1}+\zeta\right)_{n_{1}+1}\right| \cdots\left|\left(\beta_{m}+\zeta\right)_{n_{m}+1}\right|} \mid \frac{\left|\left(\beta_{1}+\zeta\right)_{s}\right|}{\left|\left(\alpha_{1}+\zeta\right)_{s}\right|} .
$$

Согласно [6, лемма 7.7, с. 108$]$ находим

$$
\left|\left(\beta_{i}+\zeta\right)_{n_{i}+1}\right| \geqslant c_{11} n_{i} ! \cdot 2^{-n_{i}} n_{i}^{-1},
$$

откуда

$$
\begin{aligned}
\left|R_{\boldsymbol{n}}(\zeta) \frac{\left(\beta_{1}+\zeta\right)_{s}}{\left(\alpha_{1}+\zeta\right)_{s}}\right| & \leqslant n_{1}^{c_{12}} \frac{\left(\left|\beta_{j}\right|+s+2\right) \cdots\left(\left|\beta_{j}\right|+s+N+1\right)}{n_{2} ! \cdots n_{m} ! s !\left(n_{1}-s\right) !} \cdot 2^{n_{1}+\cdots+n_{m}} \\
& \leqslant n_{1}^{c_{13}} \cdot \frac{2^{2 N+s} \cdot N !}{n_{1} ! \cdots n_{m} !} \cdot C_{n_{1}}^{s} \leqslant n_{1}^{c_{14}} e^{n_{1}(m \ln 4 m+\ln 4)}
\end{aligned}
$$

при допустимых значениях $j$ и $s$. Следовательно, для этих значений $j$ и $s$

$$
\left|P_{\boldsymbol{n}, j}(z)\right| \leqslant|z|^{n_{j}} n_{1}^{c_{15}} e^{n_{1}(m \ln 4 m+\ln 4)} .
$$

Пусть теперь $\alpha_{1} \neq \beta_{1}$ и $j=1,0 \leqslant s \leqslant n_{1}$. Заметим, что если $\zeta \in \Theta$, то существует положительная константа $c_{16}$, зависящая только от чисел $\alpha_{1}, \beta_{1} \ldots \beta_{m}$, такая, что $|\zeta|<n_{1}+c_{16}$. Тогда на контуре $\Theta$ имеем

$$
\begin{aligned}
\left|R_{\boldsymbol{n}}(\zeta) \frac{\left(\beta_{1}+\zeta\right)_{s}}{\left(\alpha_{1}+\zeta\right)_{s+1}}\right| & \leqslant \frac{(|\zeta|+1) \cdots(|\zeta|+N)}{\left|\left(\beta_{1}+\zeta\right)_{n_{1}+1}\right| \cdots\left|\left(\beta_{m}+\zeta\right)_{n_{m}+1}\right|} \cdot \frac{\left|\left(\beta_{1}+\zeta\right)_{s}\right|}{\left|\left(\alpha_{1}+\zeta\right)_{s+1}\right|} \\
& <n_{1}^{c_{17}} \frac{\left(n_{1}+c_{16}\right) \cdots\left(n_{1}+c_{16}+N\right)}{(s+1) !\left(n_{1}-s\right) ! n_{2} ! \cdots n_{m} !} \cdot 2^{N}<n_{1}^{c_{18}} \cdot 2^{2 N+2 n_{1}} \cdot m^{N} \\
& \leqslant n_{1}^{c_{19}} e^{n_{1}(m \ln 4 m+\ln 4)}
\end{aligned}
$$

и лемма доказана.

Определим натуральные числа $a_{1}, b_{j}, b_{i, j}, a_{1, j}(i, j=1, \ldots, m, i \neq j)$ равенствами (1). Для вычисления общего знаменателя коэффициентов многочленов $P_{\boldsymbol{n}, j}(z), 0 \leqslant$ $j \leqslant m$, нам понадобится следующая лемма, доказательство которой можно найти в [7].

ЛЕмма 5. Пусть $a, b-$ натуральные числа, $1 \leqslant a \leqslant b,(a, b)=1$. Пусть

$$
h(b)=\frac{b}{\varphi(b)} \sum_{\substack{r=1 \\(r, b)=1}}^{b} \frac{1}{r},
$$

где $\varphi-$ функиия Эйлера. Тогда для $d_{n}(b, a)=\operatorname{HOK}(a+b, a+2 b, \ldots, a+n b)$ имеет место асимптотическая формула

$$
d_{n}(b, a)=e^{h(b) \cdot n(1+o(1))} \quad \text { npu } n \rightarrow \infty .
$$


Лемма 6. Пусть

$$
\begin{gathered}
H=\sum_{k=1}^{m}\left(h\left(a_{1, k}\right)+h\left(b_{k}\right)+\sum_{\substack{l=1 \\
l \neq k}}^{m} h\left(b_{l, k}\right)\right), \\
F=2\left(\ln a_{1}+\sum_{\substack{p \mid a_{1} \\
p \text { простое }}} \frac{\ln p}{p-1}\right)+\sum_{j=1}^{m}\left(\ln b_{j}+\sum_{\substack{p \mid b_{j} \\
p \text { простое }}} \frac{\ln p}{p-1}\right)+H .
\end{gathered}
$$

Пусть $\Omega$ - наименьший общий знаменатель коэффициентов многочленов $P_{n, j}(z)$, $0 \leqslant j \leqslant m$. Тогда справедливо неравенство

$$
\Omega \leqslant e^{F n_{1}+o\left(n_{1}\right)} \quad \text { npu } n_{1} \rightarrow \infty .
$$

ДокАЗАТЕЛЬСТво. Из (13) и (14) следует, что

$$
\begin{aligned}
& \omega_{j, s}=\left.R_{n}(\zeta) \frac{\left(\beta_{1}+\zeta\right)_{s}}{\left(\alpha_{1}+\zeta\right)_{s}}\left(\beta_{j}+\zeta+s\right)\right|_{\zeta=-\beta_{j}-s}, \quad 2 \leqslant j \leqslant m, \quad 0 \leqslant s \leqslant n_{j} \\
& \quad \text { и } j=1, \quad 0 \leqslant s \leqslant n_{1} \text { в случае } \alpha_{1}=\beta_{1} ; \\
& \omega_{1, s}=\left.\left(\alpha_{1}-\beta_{1}\right) \sum_{j=1}^{m} \sum_{k=s+1}^{n_{j}} R_{n}(\zeta) \frac{\left(\beta_{1}+\zeta\right)_{s}}{\left(\alpha_{1}+\zeta\right)_{s+1}}\left(\beta_{j}+k+\zeta\right)\right|_{\zeta=-\beta_{j}-k} \\
&+\left.\left(\alpha_{1}-\beta_{1}\right) R_{\boldsymbol{n}}(\zeta) \frac{\left(\beta_{1}+\zeta\right)_{s}}{\left(\alpha_{1}+\zeta\right)_{s+1}}\left(\beta_{1}+s+\zeta\right)\right|_{\zeta=-\beta_{1}-s}, \\
& \quad 0 \leqslant s \leqslant n_{1} \text { в случае } \alpha_{1} \neq \beta_{1} .
\end{aligned}
$$

Для $\omega_{j, s}$ из (15) получаем

$$
\omega_{j, s}=\left.\frac{(\zeta-1) \cdots(\zeta-N)\left(\beta_{j}+\zeta+s\right)}{\left(\alpha_{1}+\zeta\right)_{s}\left(\beta_{1}+\zeta+s\right)_{n_{1}+1-s} \prod_{i=2}^{m}\left(\beta_{i}+\zeta\right)_{n_{i}+1}}\right|_{\zeta=-\beta_{j}-s}
$$

Для натурального числа $a$ положим

$$
\sigma_{a}^{n}=\prod_{\substack{p \mid a \\ p \text { простое }}} p^{[n /(p-1)]} .
$$

Используя обозначения (1), легко показать, что существуют положительные постоянные $c_{20}, c_{21}, c_{22}, c_{23}$, зависящие только от чисел $\alpha_{1}, \beta_{1}, \ldots, \beta_{m}$, такие, что

$$
\begin{array}{r}
\left.a_{1}^{s} \cdot \sigma_{a_{1}}^{s} \cdot d_{s}\left(a_{1, j}, c_{20}\right) \cdot \frac{(\zeta-1) \cdots(\zeta-(s-1))}{\left(\alpha_{1}+\zeta\right)_{s}}\right|_{\zeta=-\beta_{j}-s} \in \mathbb{Z}, \\
\left.b_{1}^{n_{1}+1-s} \cdot \sigma_{b_{1}}^{n_{1}+1-s} \cdot d_{n_{1}+1-s}\left(b_{1, j}, c_{21}\right) \cdot \frac{(\zeta-s) \cdots\left(\zeta-n_{1}\right)}{\left(\beta_{1}+\zeta+s\right)_{n_{1}+1-s}}\right|_{\zeta=-\beta_{j}-s} \in \mathbb{Z} .
\end{array}
$$


Для $1<i<j$

$$
\begin{aligned}
& b_{i}^{n_{i}+1} \cdot \sigma_{b_{i}}^{n_{i}+1} \cdot d_{n_{1}+1}\left(b_{i, j}, c_{22}(i)\right) \\
& \quad \times\left.\frac{\left(\zeta-n_{1}-\cdots-n_{i-1}-(i-1)\right) \cdots\left(\zeta-n_{1}-\cdots-n_{i}-(i-1)\right)}{\left(\zeta+\beta_{i}\right)_{n_{i}+1}}\right|_{\zeta=-\beta_{j}-s} \in \mathbb{Z}, \\
& b_{j}^{n_{j}} \cdot \sigma_{b_{j}}^{n_{j}} \\
& \quad \times\left.\frac{\left(\zeta-n_{1}-\cdots-n_{j-1}-(j-1)\right) \cdots\left(\zeta-n_{1}-\cdots-n_{j}-(j-2)\right)}{\left(\beta_{j}+\zeta\right)_{s}\left(\beta_{j}+\zeta+s+1\right)_{n_{j}-s}}\right|_{\zeta=-\beta_{j}-s} \in \mathbb{Z} .
\end{aligned}
$$

Для $i>j$

$$
\begin{aligned}
& b_{i}^{n_{i}+1} \cdot \sigma_{b_{i}}^{n_{i}+1} \cdot d_{n_{1}}\left(b_{i, j}, c_{23}(i)\right) \\
& \quad \times\left.\frac{\left(\zeta-n_{1}-\cdots-n_{i-1}-(i-2)\right) \cdots\left(\zeta-n_{1}-\cdots-n_{i}-(i-2)\right)}{\left(\beta_{i}+\zeta\right)_{n_{i}+1}}\right|_{\zeta=-\beta_{j}-s} \in \mathbb{Z} .
\end{aligned}
$$

Отсюда с учетом (17) и леммы 5 получаем, что

$$
\begin{gathered}
a_{1}^{n_{1}} \cdot \sigma_{a_{1}}^{n_{1}} \cdot\left(\prod_{l=1}^{m} b_{l}^{n_{l}+1} \cdot \sigma_{b_{l}}^{n_{l}+1}\right) \cdot \Lambda_{j} \cdot \omega_{j, s} \in \mathbb{Z}, \quad 2 \leqslant j \leqslant m, \quad 0 \leqslant s \leqslant n_{j} \\
\text { и } j=1, \quad 0 \leqslant s \leqslant n_{1} \text { в случае } \alpha_{1}=\beta_{1},
\end{gathered}
$$

где

$$
\Lambda_{j} \leqslant \exp \left\{\left(h\left(a_{1, j}\right)+\sum_{\substack{i=1 \\ i \neq j}}^{m} h\left(b_{i, j}\right)\right) n_{1}(1+o(1))\right\} \quad \text { при } n_{1} \rightarrow \infty .
$$

Аналогичные рассуждения для $\omega_{1, s}$ из (16) позволяют доказать, что

$$
a_{1}^{n_{1}+1} \cdot \sigma_{a_{1}}^{n_{1}+1} \cdot \prod_{l=1}^{m} b_{l}^{n_{l}+1} \cdot \Lambda \cdot \omega_{j, s} \in \mathbb{Z} \quad j=1, \ldots, m, \quad s=0, \ldots, n_{j},
$$

где

$$
\Lambda=\Lambda_{1} \cdots \Lambda_{m} \leqslant \exp \left\{\left(\sum_{l=1}^{m}\left(h\left(a_{1, l}\right)+\sum_{\substack{i=1 \\ i \neq l}}^{m} h\left(b_{i, l}\right)\right)\right) n_{1}(1+o(1))\right\} .
$$

А учитьвая то, что при $0 \leqslant s \leqslant n_{1}$

и

$$
a_{1}^{n_{1}} \cdot \sigma_{a_{1}}^{n_{1}} \cdot d_{n_{1}+1}\left(b_{1}, c_{24}\right) \cdot \frac{\left(\alpha_{1}\right)_{s}}{\left(\beta_{1}\right)_{s+1}} \in \mathbb{Z}
$$

получаем

$$
\prod_{j=2}^{m} d_{n_{1}}\left(b_{j}, c_{25}(j)\right) \cdot \sum_{\nu=1}^{s} \frac{1}{\beta_{j}+\nu} \in \mathbb{Z}
$$

$$
a_{1}^{2 n_{1}+1} \cdot \sigma_{a_{1}}^{2 n_{1}+1} \cdot\left(\prod_{l=1}^{m} b_{l}^{n_{l}+1} \cdot \sigma_{b_{l}}^{n_{l}+1}\right) \cdot \Lambda \cdot \Lambda_{0} \cdot P_{n, j}(z) \in \mathbb{Z}[z], \quad 0 \leqslant j \leqslant m
$$

где

$$
\Lambda_{0} \leqslant \exp \left\{\left(\sum_{l=1}^{m} h\left(b_{l}\right)\right) n_{1}(1+o(1))\right\} \quad \text { при } n_{1} \rightarrow \infty .
$$

Теперь из (18) следует утверждение леммы. 
ЗАмечанИЕ. Заметим, что $\omega_{j, n_{j}} \neq 0$ для каждого $j=1, \ldots, m$.

Пусть $\boldsymbol{n}_{i}=\left(n_{1}+1, \ldots, n_{m-i}+1, n_{m+1-i}, \ldots, n_{m}\right), i=0,1, \ldots, m$. Рассмотрим определитель

$$
\Delta_{\boldsymbol{n}}(z)=\left|\begin{array}{cccc}
P_{\boldsymbol{n}_{0}, 0}(z) & P_{\boldsymbol{n}_{0}, 1}(z) & \ldots & P_{\boldsymbol{n}_{0}, m}(z) \\
P_{\boldsymbol{n}_{1}, 0}(z) & P_{\boldsymbol{n}_{1}, 1}(z) & \ldots & P_{\boldsymbol{n}_{1}, m}(z) \\
\vdots & \vdots & \ddots & \vdots \\
P_{\boldsymbol{n}_{m}, 0}(z) & P_{\boldsymbol{n}_{m}, 1}(z) & \ldots & P_{\boldsymbol{n}_{m}, m}(z)
\end{array}\right| .
$$

ЛЕмма 7. Выполнено $\Delta_{\boldsymbol{n}}(z) \equiv$ const $\neq 0$.

ДокАЗАТЕЛЬСтво. Пусть $\Delta_{\boldsymbol{n}, j}(z), 0 \leqslant j \leqslant m,-$ алгебраическое дополнение элемента $P_{\boldsymbol{n}_{j}, 0}(z)$ :

$$
\Delta_{\boldsymbol{n}, j}(z)=(-1)^{j}\left|\begin{array}{ccc}
P_{\boldsymbol{n}_{0}, 1}(z) & \ldots & P_{\boldsymbol{n}_{0}, m}(z) \\
\vdots & \ddots & \vdots \\
P_{\boldsymbol{n}_{j-1}, 1}(z) & \ldots & P_{\boldsymbol{n}_{j-1}, m}(z) \\
P_{\boldsymbol{n}_{j+1}, 1}(z) & \ldots & P_{\boldsymbol{n}_{j+1}, m}(z) \\
\vdots & \ddots & \vdots \\
P_{\boldsymbol{n}_{m}, 1}(z) & \ldots & P_{\boldsymbol{n}_{m}, m}(z)
\end{array}\right| .
$$

Заметим, что $\operatorname{deg} \Delta_{n, m}(z)=N+1$ и $\operatorname{deg} \Delta_{n, j}(z)<N+1$ при $j<m$. Из леммы 2 получаем следующие равенства при $j=0,1, \ldots, m$ :

$$
P_{\boldsymbol{n}_{j}, 1}(z) f_{1}\left(\frac{1}{z}\right)+\cdots+P_{\boldsymbol{n}_{j}, m}(z) f_{m}\left(\frac{1}{z}\right)-P_{\boldsymbol{n}_{j}, 0}(z)=\frac{d_{\boldsymbol{n}_{j}}}{z^{N+1+(m-j)}}+\cdots .
$$

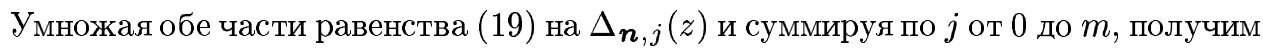

$$
\Delta_{\boldsymbol{n}}(z)=\sum_{j=0}^{m} \Delta_{\boldsymbol{n}, j}(z) \cdot P_{\boldsymbol{n}_{j}, 0}(z)= \pm d_{\boldsymbol{n}_{m}} \prod_{j=1}^{m} \rho_{j}+\frac{d_{1}}{z}+\cdots,
$$

где $\rho_{j}$ - старший коэффициент многочлена $P_{\boldsymbol{n}_{j-1}, j}$. Из последнего равенства и следует утверждение леммы, так как $d_{\boldsymbol{n}_{m}} \neq 0$.

Лемма 8. Пусть $n_{1}, \ldots, n_{m} \in \mathbb{N}, n_{1}=\max \left\{n_{1}, \ldots, n_{m}\right\}, N+1=\sum_{i=1}^{m}\left(n_{i}+1\right)$; $z=b / a, b \in \mathbb{Z}, a \in \mathbb{N},|z| \geqslant 2$. Тогда существуют положительное число $c_{26}=$ $c_{26}\left(\alpha_{1}, \beta_{1}, \ldots, \beta_{m}\right)$ и $m+1$ линейно независимых форм от чисел $1, f_{1}(a / b), \ldots, f_{m}(a / b)$ с челыми коэффичиентами,

$$
\sum_{j=1}^{m} T_{i, j} \cdot f_{j}\left(\frac{a}{b}\right)-T_{i, 0}=E_{i}, \quad i=0,1, \ldots, m,
$$

которые при $\min \left\{n_{1}, \ldots, n_{m}\right\} \geqslant c_{26}$ удовлетворяют условиям:

$$
\begin{gathered}
\left|T_{i, j}\right| \leqslant T_{j}=A_{1} \cdot e^{(F+m \ln 4 m+\ln 4) n_{1}} \cdot a^{n_{1}} \cdot\left(\frac{|b|}{a}\right)^{n_{j}}, \quad i=0,1, \ldots, m, \quad j=1, \ldots, m, \\
\left|E_{i}\right| \leqslant A_{2} \cdot e^{F n_{1}} \cdot a^{n_{1}} \cdot\left(\frac{|b|}{a}\right)^{-N}, \quad i=0,1, \ldots, m,
\end{gathered}
$$

$F$ определено в лемме $6, A_{1}=e^{o\left(n_{1}\right)}$ u $A_{2}=e^{o\left(n_{1}\right)}$ nри $n_{1} \rightarrow \infty$. 
ДокаЗАТЕльство. Требуемое утверждение следует из лемм 2-7, если положить

$$
\begin{gathered}
T_{i, j}=\Omega a^{n_{1}+1} \cdot P_{\boldsymbol{n}_{i}, j}\left(\frac{b}{a}\right), \quad i=0,1, \ldots, m, \quad j=1, \ldots, m \\
E_{i}=\Omega a^{n_{1}+1} \cdot F_{\boldsymbol{n}_{i}}\left(\frac{b}{a}\right), \quad i=0,1, \ldots, m .
\end{gathered}
$$

2. Доказательство теоремы 1 . Пусть числа $a \in \mathbb{N}$ и $b \in \mathbb{Z}$ удовлетворяют условию

$$
|b|>a^{m+1} \exp \{(m-1)(m \ln 4 m+\ln 4)+m F\} .
$$

Тогда, в частности, $|b| \geqslant 2 a$. Рассмотрим форму

$$
l=x_{1} f_{1}\left(\frac{a}{b}\right)+\cdots+x_{m} f_{m}\left(\frac{a}{b}\right)-x_{0}
$$

с цельми коэффициентами, невсе из которых равны нулю, и пусть числа $\bar{x}_{i} \geqslant \max \left(1,\left|x_{i}\right|\right)$, $i=1, \ldots, m$, таковы, что $\bar{x}_{1}=\max \left\{\bar{x}_{1}, \ldots, \bar{x}_{m}\right\}$. Определим натуральное число $n_{1}$ как наименьшее, удовлетворяющее условию

$$
\bar{x}_{1} \cdot\left(e^{m F+(m-1)(m \ln 4 m+\ln 4)} \cdot \frac{a^{m+1}}{|b|}\right)^{n_{1}}<\frac{1}{2 m} .
$$

В силу (22) при достаточно большом $\bar{x}_{1}$ такое $n_{1}$ всегда существует. Для $1<i \leqslant m$ определим $n_{i}$ как наименьшее натуральное, удовлетворяющее условию

$$
\bar{x}_{i} \cdot\left(e^{m F+(m-1)(m \ln 4 m+\ln 4)} \cdot a^{m}\right)^{n_{1}} \cdot\left(\frac{a}{|b|}\right)^{n_{i}}<\frac{1}{2 m} .
$$

Легко видеть, что при таком выборе чисел $n_{i}$ выполнено $n_{1}=\max \left\{n_{1}, \ldots, n_{m}\right\}$. Кроме того, при фиксированных $a$ и $b$ условия $\bar{x}_{1} \rightarrow \infty, n_{1} \rightarrow \infty$ и $\min \left\{n_{1}, \ldots, n_{m}\right\} \rightarrow \infty$ эквивалентны. Далее по лемме 8 среди линейных форм (20) существуют $m$ форм линейно независимых с $l$. Не ограничивая общности, пусть это формы $E_{1}, \ldots, E_{m}$. Тогда

$$
\Delta=\left|\begin{array}{cccc}
x_{0} & x_{1} & \ldots & x_{m} \\
T_{1,0} & T_{1,1} & \ldots & T_{1, m} \\
\vdots & \vdots & \ddots & \vdots \\
T_{m, 0} & T_{m, 1} & \ldots & T_{m, m}
\end{array}\right|
$$

- целое число отличное от нуля. Вычитая из первого столбца определителя остальные, умноженные соответственно на $f_{1}(a / b), \ldots, f_{m}(a / b)$, и раскладывая полученньй таким способом определитель по первому столбцу, получим неравенство

$$
1 \leqslant|\Delta| \leqslant|l| \cdot m ! \cdot T_{1} \cdots T_{m}+m ! \sum_{i=1}^{m} \frac{T_{1} \cdots T_{m}}{T_{i}} \cdot\left|E_{m}\right| .
$$

Из (23) и (21) для всех достаточно больших $n_{1}$ (т.е. для $\bar{x}_{1}>X_{0}$ ) находим

$$
\begin{aligned}
1 \leqslant & |l| \cdot e^{\left(m F+m^{2} \ln 4 m+m \ln 4\right) n_{1}} \cdot a^{m n_{1}-N} \cdot|b|^{N} \\
& +\sum_{i=1}^{m} \bar{x}_{i} e^{(m F+(m-1)(m \ln 4 m+\ln 4)) n_{1}} \cdot \frac{a^{m n_{1}+n_{i}}}{|b|^{n_{i}}} .
\end{aligned}
$$


Отсюда с учетом выбора чисел $n_{1}, \ldots, n_{m}$ при $\bar{x}_{1}>X_{0}\left(a, b, \alpha_{1}, \beta_{1}, \ldots, \beta_{m}\right)$ получаем

$$
\begin{aligned}
|l| & >\frac{1}{2} e^{-\left(m F+m^{2} \ln 4 m+m \ln 4\right) n_{1}} \cdot a^{N-m n_{1}} \cdot|b|^{-N} \\
& >C_{*}\left(\bar{x}_{1} \cdots \bar{x}_{m}\right)^{-1} \cdot\left(e^{m(m+1) F+m^{3} \ln 4 m+m^{2} \ln 4} \cdot a^{m^{2}+m}\right)^{-n_{1}} \\
& >C_{* *}\left(\bar{x}_{1} \cdots \bar{x}_{m}\right)^{-1} \cdot \bar{x}_{1}^{-\frac{m(m+1) F+m^{3} \ln 4 m+m^{2} \ln 4+m(m+1) \ln a}{\ln \mid b+1) \ln a-m F-(m-1)(m \ln 4 m+\ln 4)}} \\
& =C_{* *}\left(\bar{x}_{1} \cdots \bar{x}_{m}\right)^{-1} \cdot \bar{x}_{1}^{-\delta}
\end{aligned}
$$

где $C_{* *}=C_{* *}\left(a, b, \alpha_{1}, \beta_{1}, \ldots, \beta_{m}\right)$. Теорема доказана.

Автор выражает благодарность профессору Ю. В. Нестеренко за научное руководстBO.

\section{СПИСОК ЦИТИРОВАННОЙ ЛИТЕРАТУРЫ}

[1] Baker A. On some Diophantine inequalities involving the exponential function // Canad. J. Math. 1965. V. 17. P. 616-626.

[2] Фельдман Н. И. Об одной линейной форме // Acta Arith. 1972. V. 21. P. 347-355.

[3] Сорокин В.Н. Об иррациональности значений гипергеометрических функций // Матем. сб. 1985. Т. 127 (169). № 2 (6). С. 245-258.

[4] Хессами Пилеруд Т. Г. Оценка снизу одной линейной формы // Матем. заметки. 1999. T. 66. № 4. C. 617-623.

[5] Chudnovsky D. V., Chudnovsky G. V. Applications of Padé approximations to Diophantine inequalities in values of $G$-functions // Lecture Notes in Math. 1985. V. 1135. P. 9-51.

[6] Фельдман Н.И. Седьмая проблема Гильберта. М.: Изд-во МГУ, 1982.

[7] Alladi K., Robinson M. Legendre polynomials and irrationality // J. Reine Angew. Math. 1980. V. 318. P. 137-155.

Московский государственный университет им. М.В. Ломоносова

Поступило 08.04.1999 